\title{
ADAMTS-8 Activity in Synovial Cells in Patients with Rheumatoid Arthritis
}

\author{
Murat Baloğlu1 ${ }^{*}$, Engin Deveci² ${ }^{2}$ Şenay Deveci ${ }^{3}$ \\ ${ }^{1}$ Department of Physical Therapy and Rehabilitation, Diyarbakir Gazi Yasargil Education and Research Hospital, Diyarbakir, Turkey \\ ${ }^{2}$ Department of Histology and Embryology, School of Medicine, Dicle University, Diyarbakir, Turkey \\ ${ }^{3}$ Department of Medical Documentation, Atatürk Health Vocational School, Diyarbakir, Turkey \\ Email: *murbal21@hotmail.com, engindeveci64@gmail.com
}

How to cite this paper: Baloğlu, M., Deveci, E. and Deveci, Ș. (2020) ADAMTS-8 Activity in Synovial Cells in Patients with Rheumatoid Arthritis. Open Journal of Rheumatology and Autoimmune Diseases, 10, 125-131.

https://doi.org/10.4236/ojra.2020.103014

Received: May 16, 2020

Accepted: June 13, 2020

Published: June 16, 2020

Copyright $\odot 2020$ by author(s) and Scientific Research Publishing Inc. This work is licensed under the Creative Commons Attribution International License (CC BY 4.0).

http://creativecommons.org/licenses/by/4.0/

\section{(c) (i) Open Access}

\begin{abstract}
Rheumatoid arthritis (RA), chronic, autoimmune causing joint stiffness, pain, fatigue and functional disorders is a disease. The aim of this study is to investigate the presence of ADAMTS-8 expression in cells in synovial fluid in rheumatoid arthritis patients. Patients with active RA were aspirated with arthro synthesis from the knee joint. Blood samples were taken from the patients at the same time, plasma samples were separated. Patients with sinovial fluid treated between 2019-2020 year in the Gazi Yaşargil Hospital Physical Therapy and Rehabilitation Clinic were involved in the study that was consulted with knee pain and difficulty in performing their daily activities. Informed consent form was obtained from all of the patients (10 RA and $10 \mathrm{pa}-$ tients). CRP, RF and ESH results were statistically evaluated in patients with Control and Rheumatoid arthritis. CRP, RF and ESH results were statistically evaluated in patients with Control and Rheumatoid arthritis. In patient samples with Rheumatoid arthritis, degeneration in synovial cells was observed in some nuclei in the form of pycnosis and apoptotic. ADAMTS- 8 expression in the form of aggregates in synovial cells was found to be positive. It was observed that rheumatoid arthritis increased apoptotic change in synovial cells. ADAMTS8 in synovial cells in rheumatoid arthritis cases may cause a significant increase in apoptosis levels by suppressing some signal pathways, and predicts that apoptosis induction may play a role in the inhibition of ADAMTS8mediated synovial cell proliferation.
\end{abstract}

\section{Keywords}

Romatoid Artrit, Sinovial Fluid, ADAMTS-8

\section{Introduction}

Rheumatoid arthritis is a multisystemic, chronic and autoimmune disease that 
progresses with inflammation. Pain, fatigue, morning stiffness and limited mobility resulting from rheumatoid arthritis increase with inflammation. It also decreases the quality of life by affecting daily life, making life difficult, causing disruptions in social relations and depression. Inflammatory mediators in activated condition infiltrate synovial membranes, causing damage to bone and cartilage. Inflammatory cells are a pannus view consisting of a mixture of proliferated-laying synovial cells mixed with granulation tissue and connective tissue [1]. Some indicators are considered important in patients with rheumatoid arthritis (RA). Although serum RF, CRP level and ESH, which are among the activation indicators of RA disease, have been shown to be important, CRP in RA patients is a good indicator in the early stages of erosion development in the joints [2].

ADAMTS-8 has cell adhesion, cell fusion, proteolysis and signal transduction functions [3]. This protease has been shown to separate proteoglycans such as aggrecan, versican and brevican [4]. The substrate aggregate ADAMTS- 8 is expressed in macrophage-rich regions in atherosclerosis [5]. The aim of this study is to investigate the presence of ADAMTS-8 expression in cells in synovial fluid in rheumatoid arthritis patients.

\section{Material and Method}

Patients with active RA were aspirated with arthro synthesis from the knee joint. Blood samples were taken from the patients at the same time, plasma samples were separated. Ethics committee approval was received. Gazi Yaşargil Training and Research Hospital Physical Therapy and Rehabilitation outpatient clinic 20192020 ACR 2010 ACR/Eular criteria monitored in according to the patients diagnosed with RA were taken. Retrospective demographic characteristics, physical examination findings and laboratory data from patients' files ESH, CRP, RF, levels were measured from the data. Informed consent form was obtained from all of the patients (10 RA and control 10 patients). With each patient in the supine position, a minimum of $5 \mathrm{~mL}$ of synovial fluid was aspirated from the lateral suprapatellar pouch of the affected knee via an injector. Blood samples were also collected. The synovial fluid was subjected to high-speed centrifugation, the supernatant was discarded, and $10 \%$. (v/v) neutral formalin was added. (pellet: formalin volume ratio, 1:3). After $2 \mathrm{~h}$ of fixation, the clear bright supernatant was discarded and the tube was inverted on filter paper to allow excess fluid to drain. The pellet was then placed on filter paper using a spatula and eosin stain solution was added using a Pasteur pipette. After the cell aggregate developed a red colour, it was wrapped in filter paper and placed in a cassette, which was then stored in a fixation box for tissue processing. The cells were examined following Harris haematoxylin and eosin (H\&E) staining. The synovial fluid was also immunostained and examined under a light microscope.

\section{Immunohistochemical Staining}

Sections for immunohistochemical analysis were placed in distilled water and 
washed three times for 5 min with phosphate-buffered saline. (PBS) Antigen retrieval was performed in a microwave oven. (Bosch, $700 \mathrm{~W}$ ) for $3 \mathrm{~min}$ at $90^{\circ} \mathrm{C}$ in citrate buffer ( $\mathrm{pH} \mathrm{6)}$. The sections were washed three times for 5 min with PBS and incubated with hydrogen peroxide for $20 \mathrm{~min}$. The sections were washed 3 times for 5 min with PBS and blocked with Ultra V Block. (lot PHL150128; Thermo Fisher Scientific) for $8 \mathrm{~min}$. After draining, primary antibodies were directly added to the sections. The antibody were ADAMTS-8 (1/100) followed by incubation overnight at $4^{\circ} \mathrm{C}$. The sections were washed 3 times for 5 min with PBS and incubated with biotinylated secondary antibody for $14 \mathrm{~min}$. After washing with PBS, streptavidin peroxidase was added for $15 \mathrm{~min}$ followed by washing 3 times for 5 min with PBS and the addition of DAB for up to $10 \mathrm{~min}$. As the reaction developed, the slides were placed in PBS, counterstained with Harris haematoxylin. (Hematoxylene and Eosin, H\&E) for $45 \mathrm{~s}$, dehydrated through baths of ascendingalcohol proportions cleared in xylene mountedwith Entellan and examined under the light microscope (Carl Zeizz, Germany).

\section{Biochemical Analysis}

ESR was determined by Westergen method while CRP was measured with nephelometry. (Behring 100, Behring, Germany). Anti CCP IgG autoantibodies were detected with ELISA method. (Euroimmun, Germany). Disease activity score was measured with Disease Activity Score 28-erythrocyte sedimentation rate (DAS28-ESR) criteria.

\section{Statistical Analysis}

CRP, RF and ESH results were statistically evaluated in patients with Control and Rheumatoid arthritis. Statistical significance was subjected to the independent $\mathrm{t}$ test. Results were presented as mean $\pm \mathrm{SD}$ and $\mathrm{p}<0.05$ was considered significant.

\section{Result and Discussion}

Basic demographic data of the two groups should be listed out and compared. The amount of CRP was $3.36 \pm 2.47$ in the control group and $17.41 \pm 6.57$ in the RA group.

It was concluded that RF and ESH values were $13.45 \pm 3.54$ in the control group and $35.94 \pm 8.90$ and $39.50 \pm 11.48$ in the $12.80 \pm 3.33$ RA group. Compared to the control group, $\mathrm{CRP}, \mathrm{RF}$ and ESH results were statistically evaluated in patients with Control and Rheumatoid arthritis. .

Basic demographic data of the two groups should be listed out and compared. It is shown in Table 1 and Figure 1.

Histopathologic results (Figure 2).

İmmunohistochemical results (Figure 3).

In addition, positive expression of ADAMTS- 8 in plasma cells and some leukocyte cells was evaluated as an indicator of inflammation. 
Table 1. Statistical results of CRP, RF and ESH measurements of control and Rheumatoid arthritis groups.

\begin{tabular}{cccc}
\hline & Control & Rheumatoid arthritis & \multirow{2}{*}{ p value } \\
\cline { 1 - 2 } CRP & $3.36 \pm 2.47$ & mean \pm SD & \\
RF & $13.45 \pm 3.54$ & $35.94 \pm 8.90$ & $\mathrm{p}<0.01$ \\
ESH & $12.80 \pm 3.33$ & $39.50 \pm 11.48$ & \\
\hline
\end{tabular}



Control

Rheumatoid arthritis

Figure 1. Graphical view of CRP, RF and ESH analysis in control and RA groups. ${ }^{\star}$ Sign indicates high values in patients with rheumatoid arthritis according to control.

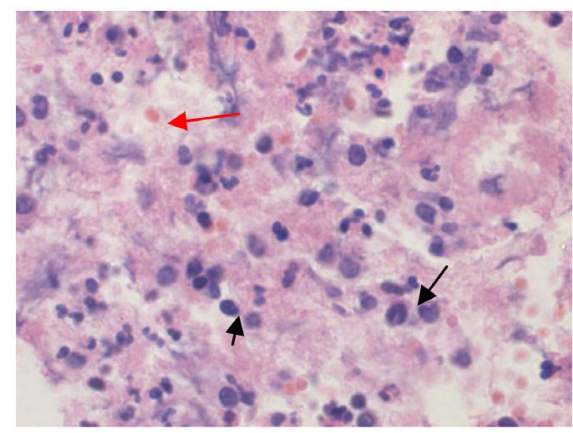

(a)

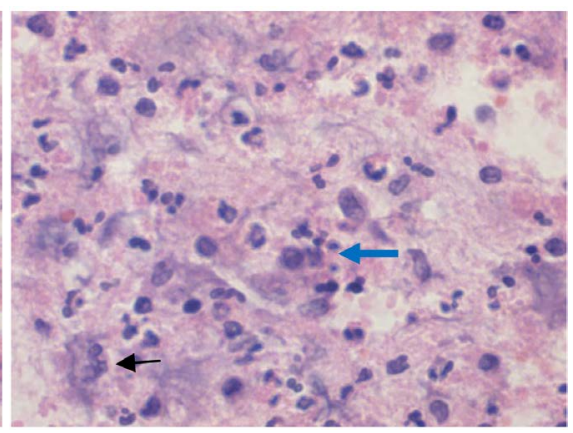

(b)

Figure 2. (a) Control group: Synovial cells rich in nucleus flat chromatin in the joint fluid and plasma cells solitaryly distributed were fibroblast and macrophage cells (arrow). Extrasellular matrix density was evident, and a small number of lymphocytes, granular loci and erythrocyte cells (red arrow) were seen in this area (a). (b) In patient samples with rheumatoid arthritis, degeneration in synovial cells (arrow) was observed in some nuclei in the form of pycnosis and apoptotic. It was observed that erythrocytes diffuse diffusely with hypertrophy, a large number of neutraphil cells (blue arrow) in macrophage cells (b).

Rheumatoid arthritis inflammatory cell infiltration, synovial. It is a chronic disease characterized by hyperplasia in tissue and a destruction of cartilage and bone. Rheumatoid arthritis becomes chronic and causes early mortality and high morbidity. Acute inflammation often indicates a sudden onset; with classic symptoms like heat, pain, redness, and swelling, it becomes visible within minutes or hours. 


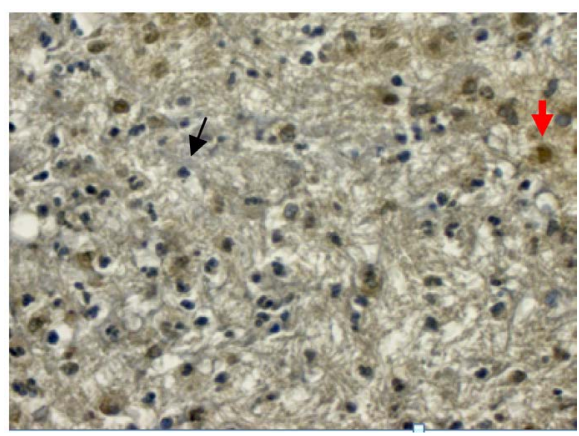

(a)

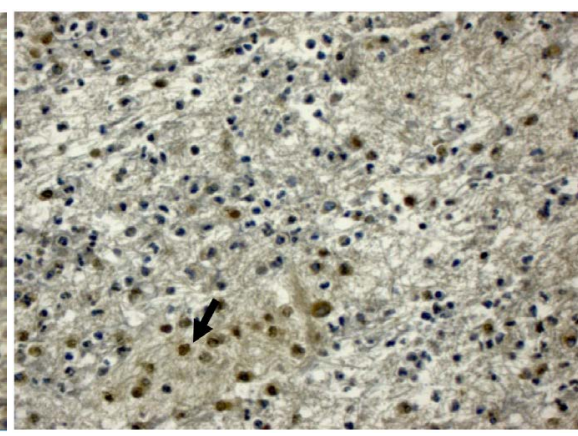

(b)

Figure 3. (a) Control group: Negative ADAMTS-8 expression was evident in synovial cell (arrow) and connective tissue cells in control group sections. Expression of ADAMTS-8 was positive in some macrophage cells (red arrow). (b) In the rheumatoid arthritis group, ADAMTS-8 expression in the form of aggregates in synovial cells was found to be positive. It was observed that rheumatoid arthritis increased apoptotic change in synovial cells (arrow).

Chronic inflammation develops over a long period of time and can last for days, weeks or months. Neutrophils are the most common inflammatory cells in acute synovitis. In chronic synovitis due to osteoarthritis, lymphocytic infiltrates are often found in large quantities, together with lymphocytic infiltrates [6]. In our study, in the control group sections, the synovial cells had nuclei-rich nuclei and fibroblasts, macrophages and few erythrocyte cells in the extracellular matrix (Figure 2(a)). In histopathological examination of rheumatoid arthritis patients, synovial cells degeneration, pycnotic nucleus and apoptosis were observed (Figure 2(b)).

Aging can lead to inadequate removal of proteins that have been exposed to mitochondrial dysfunction and oxidative damage in chondrocytes. This event may trigger cell aging and an increase in the production of inflammatory cytokines [7].

ADAMTS enzymes, known as enzymes responsible for cartilage matrix rupture in diseases such as osteoarthritis and rheumatoid arthritis, are a family of proteinases that play critical roles in the reconstruction, coagulation, morphogenesis, ovulation, angiogenesis and central nervous system diseases [3].

ADAMTS-8 protein has an angiogenetic inhibitory effect in the structure of the extracellular matrix in the regulation of various cancers that have the ability to suppress tumor genes in tumor proliferation [8]. ADAMTS-8 is a large molecule that carries an enzyme that binds to lipoproteins from proteoglycans in the extracellular matrix to versican cytokines. It has been reported that ADAMTS-8 protein may be under the influence of extracellular matrix damage due to diabetes and changes in the basement membrane structure [9]. Kaya $S$ et al. [10] stated that the inflammatory process in diabetic rats may increase cytokine activity due to the change in the vertical molecule structure in ADAMTS-8.

Some analyzes ADAMTS and the role of these MMP-like activities in human cartilage are markedly different. Excessive ADAMTS activity in vivo is destruc- 
tive for the cartilage matrix because most of the products carrying glycosaminoglycan. (GAG) release from tissue into synovial fluid. MMP-like activity has been reported to be non-destructive, as it is retained in tissue following cleavages in the region [11]. In control group sections, ADAMTS-8 expression was negative in most synovial cells, whereas ADAMTS- 8 expression was positive in macrophage cells (Figure 3(a)). We observed that the expression of ADAMTS8 in synovial cells in rheumatoid arthritis cases may cause a significant increase in apoptosis levels by suppressing some signal pathways, and predicts that apoptosis induction may play a role in the inhibition of ADAMTS8-mediated synovial cell proliferation (Figure3(b)). It is thought that synovial cells can affect cartilage matrix and cartilage tension and induce enzymes that degrade matrix. Increased inflammation in joint fluid in cases of rheumatoid arthritis is thought to cause disruption in the cartilage matrix and apoptotic cell development.

\section{Conflicts of Interest}

The authors declare no conflicts of interest regarding the publication of this paper.

\section{References}

[1] Mitchell, R.N. (2003) Immune Disorders. In: Erez, S., Ed., 7th Edition, Robbins Basic Pathology Nobel Medical Bookstore, Istanbul, 103-164.

[2] Kiener, H.P., Baghestanian, M., Dominkus, M., Walchshofer, S., Ghannadan, M., Willheim, M., et al. (1998) Expression of the C5a Receptor (CD88) on Synovial Mast Cells in Patients with Rheumatoid Arthritis. Arthritis Rheum, 41, 233-245. https://doi.org/10.1002/1529-0131(199802)41:2<233::AID-ART7>3.0.CO;2-V

[3] Porter, S., Ian, M., et al. (2005) The ADAMTS Metalloproteinases. Biochemical Journal, 386, 15-27. https://doi.org/10.1042/BJ20040424

[4] Yamaji, N., Nishimura, K., et al., (2001) Novel Metalloprotease Having Aggrecanase Activity. Yamanouchi Pharmaceutical, Japan.

[5] Lingen, M.W. (2001) Role of Leukocytes and Endothelial Cells in the Development of Angiogenesis in Inflammation and Wound Healing. Archives of Pathology \& Laboratory Medicine, 125, 67-71.

[6] Berenbaum, F. (2013) Osteoarthritis as an Inflammatory Disease. Osteoarthritis and Cartilage, 21, 16-21.

[7] Tortorella, M.D., Malfait, F., Barve, R.A., Shieh, H.S. and Malfait, A.M. (2009) A Review of the ADAMTS Family Pharmaceutical Targets of the Future. Current Pharmaceutical Design, 15, 2359-2374. https://doi.org/10.2174/138161209788682433

[8] Kılıç, Ö.M., Aynekin, B., et al. (2017) Differentially Regulated ADAMTS1, 8, 9, and 18 in Pancreas Adenocarcinoma. Przegląd Gastroenterologiczny, 12, 262-270. https://doi.org/10.5114/pg.2017.72101

[9] Zhao, C., Zya, Y., et al. (2011) The Quantification of ADAMTS-4 and 8 Expression and Selection of Reference Genes for Quantitative Real-Time PCR Analysis in Myocardial Infarction. Biomedicine \& Pharmacotherapy, 65, 555-559. https://doi.org/10.1016/j.biopha.2010.12.002

[10] Kaya, S., Nergiz, Y., Asır, F., Gokalp-Ozkorkmaz, E., Erdogan, G. and Deveci, E. 
(2019) Immunohistochemical Analysis of Sflt-1 of Expression Diabetic Rat Testis Tissue. International Journal of Health Services Research and Policy, 4, 225-232. https://doi.org/10.23884/ijhsrp.2019.4.3.07

[11] Sandy, J.D. and Verscharen, C. (2001) Analysis of Aggrecan in Human Knee Cartilage and Synovial Fluid Indicates That Aggrecanase (ADAMTS) Activity Is Responsible for the Catabolic Turnover and Loss of Whole Aggrecan Whereas Other Protease Activity Is Required for C-Terminal Processing in Vivo. The Biochemical Journal, 358, 615-626. https://doi.org/10.1042/bj3580615 